\title{
Comportamento sob Fluência em Elastômeros Termoplásticos Vulcanizados Baseados em Poliamida 6 e Borracha Nitrílica
}

\author{
Ana C. 0. Gomes, Bluma G. Soares \\ Instituto de Macromoléculas Professora Eloisa Mano, UFRJ \\ Marcia G. Oliveira \\ Divisão de Processamento e Caracterização de Materiais, INT \\ Caio M. Paranhos \\ Departamento de Engenharia de Materiais, UFSCar
}

Resumo: Os materiais testados neste trabalho são o resultado de um estudo do uso de aditivos e compatibilizantes na mistura de poliamida 6 (PA6) e borracha nitrílica (NBR), realizado com o objetivo de melhorar suas propriedades mecânicas e facilitar o processamento da mistura. Fluência ("creep") é um teste mecânico importante ao simular a aplicação final do material de engenharia, possibilitando a previsão do desempenho de modo comparativo. Entretanto, é um teste pouco explorado na caracterização de TPV's. A melhora nas propriedades com a adição de aditivos e a eficiência do processo de compatibilização pode ser observada através da variação na compliância das amostras analisadas. Os resultados são correlacionados usando testes de densidade, teor de gel, resistência à tração e microscopia eletrônica de varredura. O presente trabalho mostra que é possível avaliar um material em condições semelhantes à aplicação final em um teste rápido e com gasto mínimo de material.

Palavras-chave: PA6, NBR, TPV, propriedades mecânicas, fluência.

\section{Creep Behavior of Polyamide 6/Nitrylic Rubber TPV's Blends}

\begin{abstract}
The materials tested in this work are the result of a study involving the use of additives and compatibilization in blends of PA6 and NBR, which was aimed at enhancing the mechanical properties and processability of the blend. Creep is an important mechanical test since it simulates the final application of the material, allowing a prediction of material performance, in a comparative way. However, this is a test seldom explored in the characterization of TPV's. The enhancement of the properties induced by additives and the efficiency of compatibilization process can be observed through the analysis of changes in the compliance of the samples. The results are correlated using measurements of density, gel content, tension strength and scanning electron microscopy. The present work shows it to be possible to evaluate a material under conditions similar to those in the final applications, in a fast test and with minimal material waste.
\end{abstract}

Keywords: PA6, NBR, TPV, mechanical properties, creep.

\section{Introdução}

Elastômeros termoplásticos vulcanizados ("thermoplastic elastomers vulcanized" - TPV) são materiais altamente promissores devido às suas características de multi-aplicabilidade e reprocessabilidade ${ }^{[1,2]}$. TPV's unem a processabilidade dos termoplásticos com a elasticidade dos elastômeros em um único material. Dentre os vários tipos de TPV, a mistura polimérica tem maior potencial de aplicabilidade devido à ampla gama de materiais que podem ser combinados. O meio mais comum de se obter um TPV através de misturas é a vulcanização dinâmica da fase elastomérica, descrita pela primeira vez por Gessler e colaboradores ${ }^{[3]}$.

Poliamidas são termoplásticos com elevado módulo, resistência mecânica, estabilidade dimensional a tempera- turas elevadas e resistência química. Borracha nitrílica, por sua vez, é um elastômero para propósitos especiais, devido à sua ótima resistência a óleo e a abrasãa ${ }^{[4-7]}$. Supõe-se que a combinação de tais componentes pode gerar um material com boa resistência a óleo quente e boas propriedades mecânicas, particularmente a altas temperaturas ${ }^{[8,9]}$. Apesar do potencial, pouco se tem publicado em relação a misturas de PA6 e NBR, provavelmente devido à alta temperatura necessária para o processamento e moldagem do PA6, que pode causar a degradação do elastômero. Nosso grupo de pesquisa estudou a incorporação de aditivos a fim de melhorar a processabilidade e as propriedades finais da mistura ${ }^{[10]}$.

O teste de fluência ("creep") é um ensaio mecânico importante ao simular deformações que podem ocorrer duran-

Autor para correspondência: Ana C. O. Gomes, Instituto de Macromoléculas Profa. Eloísa Mano, UFRJ, Av. Horácio Macedo 2030, Bloco J, Laboratório J112, Centro de Tecnologia, Cidade Universitária, CEP: 21945-598, Rio de Janeiro, RJ, Brasil. E-mail: acogomes@ima.ufrj.br 
te a aplicação final do material, sendo capaz de prever o desempenho do material em função do tempo ${ }^{[11,12]}$. Os testes de fluência são especialmente úteis para estudar materiais sob taxas de cisalhamento muito baixas ou em baixa freqüência, avaliando não só o material, mas o projeto do $\operatorname{artefato}^{[13]}$.

$\mathrm{O}$ teste de fluência consiste em duas etapas. Na primeira, uma tensão pré-determinada é aplicada à amostra, e a deformação resultante é registrada em função do tempo. Na segunda etapa, a tensão é retirada, e mede-se a deformação recuperável, ou seja, o retorno da deformação resultante da resposta elástica do material. A Figura 1 representa o comportamento esperado de um material viscoelástico, semelhante aos ensaiados nesse trabalho, durante o ensaio descrito.

A fluência está intimamente relacionada ao envelhecimento físico, ou seja, a danos causados pela lenta relaxação estrutural das cadeias poliméricas. Esse envelhecimento é diferente de fenômenos de degradação, por ser termodinamicamente reversível. $\mathrm{O}$ ensaio não pode ser diretamente relacionado ao envelhecimento físico do material, mas a comparação do comportamento de fluência pode ser utilizada como um bom parâmetro de avaliação ${ }^{[11]}$. O conhecimento insuficiente de como o material flui durante a solicitação gerada pela aplicação pode levar a falhas consideráveis do produto final. Essa análise é importante especialmente para elastômeros, que passam por fases de composição antes da moldagem ${ }^{[14]}$. Estudos de fluência em tempos curtos podem, ainda, dar informações sobre a interação interfacial em materiais heterogêneos, como as misturas poliméricas ${ }^{[15]}$. Apesar de comprovada utilidade no estudo de termoplásticos reforçados ou elastômeros vulcanizados ${ }^{[16]}$, muito pouco existe na literatura sobre comportamento de fluência em TPV's, materiais que unem propriedades de ambos.

O presente trabalho tem o objetivo de avaliar o efeito do uso de aditivos e agentes compatibilizantes no comportamento sob fluência de misturas poliméricas baseadas em PA6 e NBR. Os aditivos são adicionados em um sistema de "masterbatch" - MB, essencial para o total aproveitamento dos efeitos sinergísticos envolvidos, e muito pouco explorado no preparo de TPV' ${ }^{[10]}$. Os resultados acompanham a evolução do material no sentido da obtenção de um TPV dimensional-

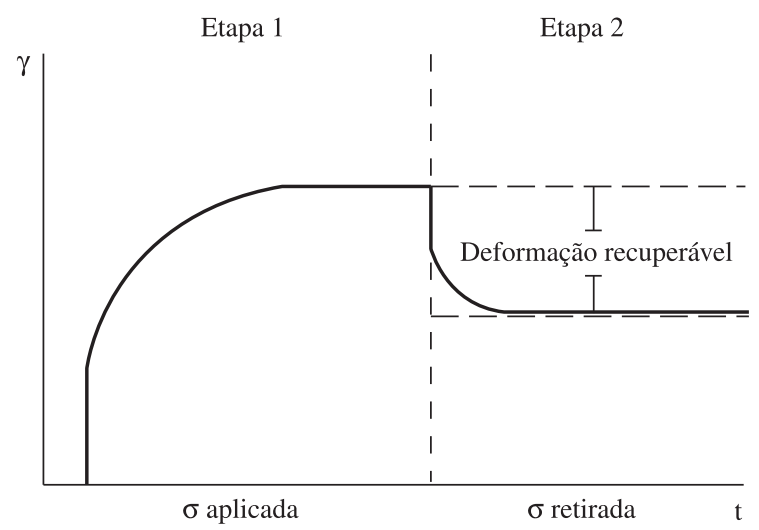

Figura 1. Representação da resposta de um material viscoelástico durante o ensaio de fluência. mente estável e de possível aplicabilidade em condições mais severas que a maioria dos materiais disponíveis no mercado.

\section{Experimental}

\section{Materiais}

NBR $(28 \%$ p/p de acrilonitrila; viscosidade Mooney $=60$, a $100{ }^{\circ} \mathrm{C}$ ), NBR carboxilada (NBRCOOH $-28 \% \mathrm{p} / \mathrm{p}$ de acrilonitrila, $12 \%$ p/p de grupos carboxila) e resina fenólica SP 1045 (RF), foram doados por Petroflex Ind e Com. PA6 (Radilon $\mathrm{S}^{\circledR}$ natural, índice de fluidez $=19,6 \mathrm{~g} / 10 \mathrm{~min}$ ASTM 1238D, densidade $1,14 \mathrm{~g} / \mathrm{cm}^{3}$ ) foi gentilmente cedido por Radici Group. Copolímero de etileno-acetato de vinila modificado com anidrido maleico (EVAMA - teor de acetato de vinila de $28 \%$, teor de MA de $0,8 \%$ p/p, índice de fluidez de $16 \mathrm{~g} / 10 \mathrm{~min}$, densidade $0,95 \mathrm{~g} / \mathrm{cm}^{3}$ ) foi adquirido de Proquimil Produtos Químicos Ltda. O antioxidante para a fase NBR, Naugard $445^{\circledR}$ (uma difenilamina complexa), foi gentilmente cedido por Crompton Corporation do Brasil. Os antioxidantes para fase PA6, Irganox $1010^{\circledR}$ (composto fenólico) e Irgafos $168^{\circledR}$ (um fosfito) foram adquiridos da Ciba Corporation do Brasil. Cloreto estanoso $\left(\mathrm{SnCl}_{2}\right)$ foi adquirido comercialmente e usado como recebido. NBR-oxazolina e NBR-epóxi (ambas com aproximadamente $3 \mathrm{mmol.g} \mathrm{g}^{-1}$ de grupos funcionais) foram obtidas em nosso laboratório através da modificação da NBR, conforme descrito por Almeida ${ }^{[17]} \mathrm{e}$ Rocha $^{[18]}$, respectivamente.

\section{Preparação das misturas}

Todas as amostras foram preparadas em um misturador de câmara interna acoplado a um reômetro de torque Brabender Plasticorder Rheometer, com rotor tipo "banbury", a $80 \mathrm{rpm}$ de velocidade. O MB de PA6 (MBPA) foi preparado a $240{ }^{\circ} \mathrm{C}$, e o MB de NBR (MBNBR) a $40{ }^{\circ} \mathrm{C}$, ambos processados por 3 minutos. Todas as misturas foram preparadas misturandose os MBs de PA e NBR na proporção 50:50, a $220^{\circ} \mathrm{C}$, durante 9 minutos. O MBPA foi previamente aquecido, no interior da câmara de mistura, por 2 minutos. Após esse tempo, a mistura é iniciada, o MBPA é processado por 1 minuto, e os componentes da mistura adicionados na seguinte ordem: MBNBR (2 minutos), compatibilizante (2 minutos), sistema de reticulação, e Naugard (adicionado no último minuto de mistura). A composição das misturas realizadas é descrita na Tabela 1. Todos os materiais foram secos em estufa a vácuo a $100{ }^{\circ} \mathrm{C}$ antes de cada etapa de processamento.

\section{Extração da fase NBR não reticulada}

Amostras retangulares com aproximadamente $10 \mathrm{~mm} \times$ $10 \mathrm{~mm} \times 2 \mathrm{~mm}$ foram extraídas em sistema Soxhlet, com tolueno, durante 24 horas. 
Tabela 1. Composição de amostras analisadas.

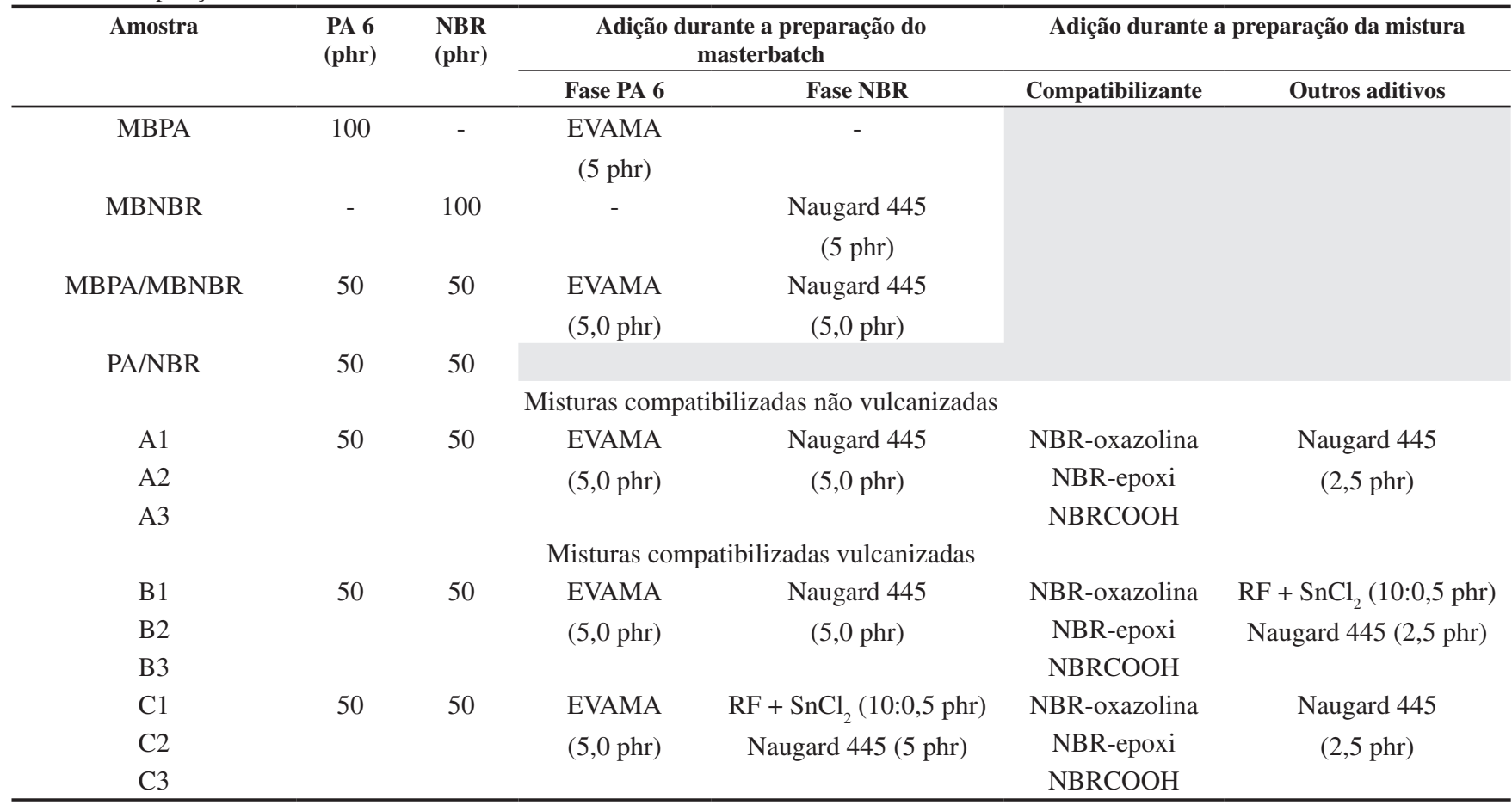

phr: "parts per hundred of rubber" - partes por 100 partes de borracha.

\section{Determinação de densidade relativa}

As densidades de cada amostra foram determinadas de acordo com a norma ASTM D 297, em álcool etílico P.A., a temperatura ambiente.

\section{Ensaio de resistência à tração}

Os corpos de prova foram obtidos em uma injetora semiindustrial Battenfeld Plus 35 com 110 bar de pressão de molde, $240{ }^{\circ} \mathrm{C}$ de temperatura de injeção e 80 bar de pressão de injeção. As medidas dos corpos moldados seguem a norma DIN53504. O ensaio foi realizado a temperatura e umidade ambiente em máquina de ensaios universal EMIC DL-2000, com $200 \mathrm{~mm} / \mathrm{min}$ de velocidade.

\section{Ensaios de fluência}

As amostras foram prensadas em filmes de aproximadamente $0,1 \mathrm{~mm}$ de espessura, em prensa hidráulica Carver, a $240{ }^{\circ} \mathrm{C}$, por 1 minuto, com 2,5 t de pressão. Os corpos de prova de fluência foram cortados dos filmes obtidos, em forma de retângulo com $6 \mathrm{~mm} \times 30 \mathrm{~mm}$. Os ensaios foram realizados em DMA Q800, da TA Instruments, em garra de tração em filme. As condições utilizadas foram: temperatura de $25{ }^{\circ} \mathrm{C}$, tempo de estabilização de temperatura de 5 minutos, aplicação instantânea de tensão de $0,1 \mathrm{MPa}$, sustentação de tensão por 30 minutos, retirada instantânea da tensão aplicada, recuperação de deformação por 60 minutos.

\section{Microscopia eletrônica de varredura}

As imagens de Microscopia Eletrônica de Varredura (MEV) foram obtidas em um equipamento JEOL $5610 \mathrm{LV}$ com voltagem de $30 \mathrm{kV}$. As amostras foram criofraturadas após 2 horas de imersão em nitrogênio líquido, e posteriormente extraídas seletivamente com tolueno. As amostras foram recobertas com ouro para uso do detector de elétrons secundários.

\section{Resultados e Discussão}

Inicialmente, é preciso esclarecer que o ensaio de fluência foi realizado em condições de não-lineariedade, aplicando-se uma tensão inicial elevada em relação às dimensões do filme. A escolha de um ensaio em condições não-lineares está baseada principalmente na correspondência com uma condição de aplicação real, onde o artefato final dificilmente será submetido a solicitações mecânicas mínimas ${ }^{[12]}$.

A amostra de poliamida pura (PA6) mostra um comportamento bastante peculiar, apresentando deformação "negativa", o que é fisicamente incoerente. Este comportamento pode ser associado à instabilidade dimensional do corpo de prova obtido através do corte de filme prensado, um efeito da "memória" das moléculas poliméricas. É importante lembrar que as amostras são filmes finos obtidos através de prensagem, moldagem obtida através da deformação bidimensional do material e reconhecidamente produtora de tensões residuais no material moldado. Como pode ser observado na Figura 2, uma vez que a força inicial é aplicada, o corpo deforma-se pouco, mas a mobilidade iniciada pela tração permite uma relaxação da conformação das moléculas, fazendo com que o filme "encolha". No momento em que a força é retirada (em 30 minutos de análise), a deformação inicial é 


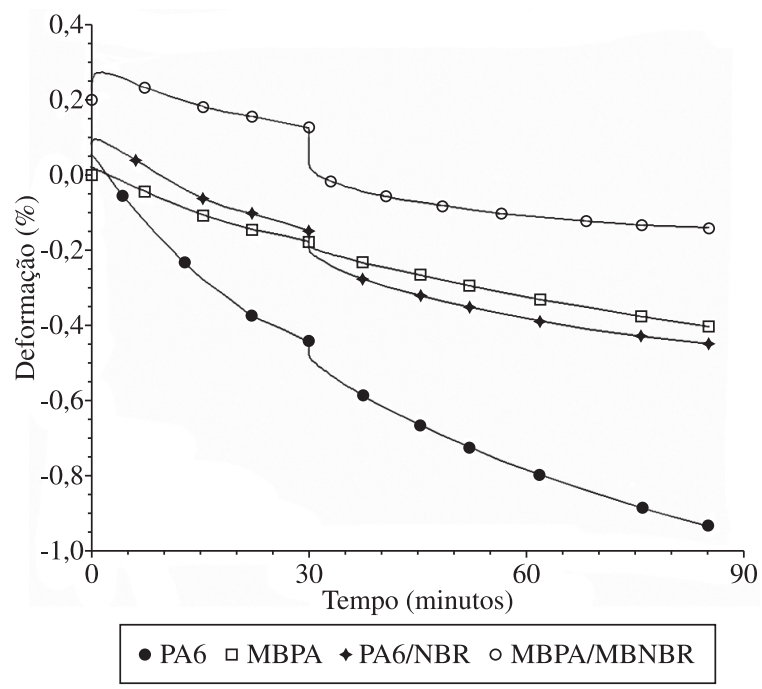

Figura 2. Perfil de deformação das amostras aditivadas.

recuperada, mas a relaxação resultante da memória do material se mantém, e o filme continua a encolher. De fato, alguns autores têm considerado que a região amorfa de um polímero semi-cristalino pode não estar em um estado completamente relaxado, mesmo em temperaturas próximas à $\mathrm{T}_{\mathrm{g}}$, devido à restrição causada pelos domínios cristalinos ${ }^{[19]}$.

\section{Efeito da mistura e da aditivação}

Observa-se o comportamento de relaxação descrito acima, em outras amostras, sendo que a instabilidade dimensional diminui gradualmente à medida que os aditivos e a própria NBR são adicionados ao material. Ainda na Figura 2, observa-se que a amostra MBPA relaxa bem menos que a PA6 pura, apresentando uma redução mais discreta na deformação. A mistura sem aditivos (PA/NBR) tem uma deformação inicial um pouco maior, o que é esperado, devido à presença da $\mathrm{NBR}^{[16]}$. PA/NBR flui consideravelmente ao longo do tempo, apresentando uma relaxação mais acentuada que o MBPA. A mistura aditivada (MBPA/MBNBR) apresenta menor relaxação que PA/NBR, porém maior deformação na aplicação da tensão inicial.

O comportamento apresentado pela mistura MBPA/ MBNBR pode estar ocorrendo devido ao efeito estabilizador do EVAMA. O grupo funcional anidrido maleico no EVAMA é capaz de reagir com o grupo terminal amina da PA 6. A reação é amplamente descrita na literatura, e frequentemente associada a melhorias em propriedades mecânicas de misturas poliméricas, com destaque para tensão de escoamento ${ }^{[20]}$. As moléculas geradas pela reação são diferentes da fase cristalizável da mistura e tendem a ser excluídas da estrutura organizada, durante o processo de cristalização. Se o fenômeno de relaxação ocorre devido à instabilidade da fase amorfa do PA 6, como citado anteriormente, é razoável que o produto da reação entre EVAMA e PA6, localizado na fase amorfa, esteja agindo no sentido de diminuir esse efeito.

\section{Efeito da compatibilização}

A Figura 3 apresenta o perfil de deformação de amostras compatibilizadas, vulcanizadas ou não. A amostra não vulcanizada (A1) comporta-se de modo semelhante à mistura vulcanizada dos MBs (MBPA/MBNBR). Essa semelhança indica que o processo de compatibilização com grupos 2-oxazolina resultou em um perfil de resistência a fluência semelhante ao processo de formação das ligações cruzadas. Ou seja, as ligações químicas resultantes do processo de compatibilização com 2-oxazolina geraram resistência semelhante a reticulação da fase elastomérica. As reações de grupos 2-oxazolina com ambos os grupos funcionais terminais da PA6 são amplamente discutidas e descritas na literatura $^{[17,21]}$.

O uso de grupos epóxi na compatibilização (A2) resulta em uma grande deformação inicial, com pouca recuperação de deformação. Apesar de gerar um perfil elastomérico, as ligações formadas pelo grupo epoxi não resultaram na mesma resistência gerada com grupos 2-oxazolina. A NBR-caboxilada (A3) resultou em baixíssima deformação e boa recuperação de deformação, obtendo ainda, boa estabilização do fenômeno de relaxação do corpo de prova, sem a necessidade da vulcanização.

\section{Influência da vulcanização dinâmica}

As amostras não vulcanizadas apresentam, como pode ser observado na Figura 3, grande fluência e efeito de relaxação de conformação, devido à fase elastomérica não reticulada. O processo de vulcanização dinâmica causou aumento na deformação em todas as amostras, que apresentam um bom perfil elastomérico. B1 e B2 (amostras com sistema de reticulação adicionado durante o processamento, com NBR-oxazolina e NBR-epóxi como compatibilizantes, respectivamente) aumentaram a recuperação da deformação com a vulcanização, enquanto que B3 (com NBR-carboxi-

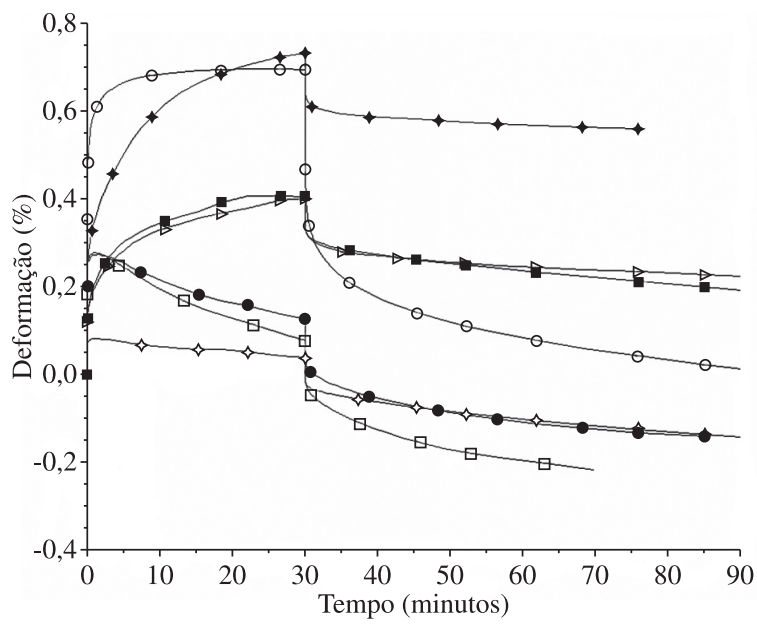

- $\mathrm{MBPA} / \mathrm{MBNBR} \square \mathrm{A} 1+\mathrm{A} 2 \diamond \mathrm{A} 3 \backsim \mathrm{B} 1 \circ \mathrm{B} 2 \triangleright \mathrm{B} 3$

Figura 3. Perfil de deformação de misturas compatibilizadas, não vulcanizadas e vulcanizadas com adição do sistema de vulcanização durante o processamento. 
lada como compatibilizante) apresentou uma recuperação de deformação baixa. A recuperação de deformação é frequentemente associada à melhor interação entre as fases da mistura $^{[14,16,19,22-24]}$.

É possível que o aumento de tensão interfacial gerada pela reticulação tenha anulado o efeito da compatibilização no caso do uso de NBR-carboxilada. Essa hipótese pode ser apoiada pelo fato das ligações químicas geradas pelos grupos epóxi e 2-oxazolina serem mais fortes que as geradas pelo grupo carboxila ${ }^{[16]}$. A literatura relata casos onde os efeitos benéficos da melhor dispersão de domínios são superados pela má interação interfacial ${ }^{[22]}$.

\section{Influência do modo de vulcanização}

A Figura 4 apresenta a comparação entre dois métodos de vulcanização utilizados. A adição do sistema de vulcanização no MBNBR (amostras C) resulta em processo de vulcanização mais eficiente, e o efeito de relaxação desaparece devido à estabilidade obtida pela reticulação da fase elastomérica. Essas amostras geram pouca resistência à deformação inicial, com recuperação incompleta da deformação. É possível observar, ainda, que a amostra $\mathrm{C} 1$ entra em equilíbrio rapidamente, gerando uma região "achatada" no gráfico, típico de amostras reticuladas ${ }^{[13]}$. A adição do sistema de vulcanização durante o processamento (amostras B) resulta em menor deformação inicial, porém apresenta menor recuperação da deformação.

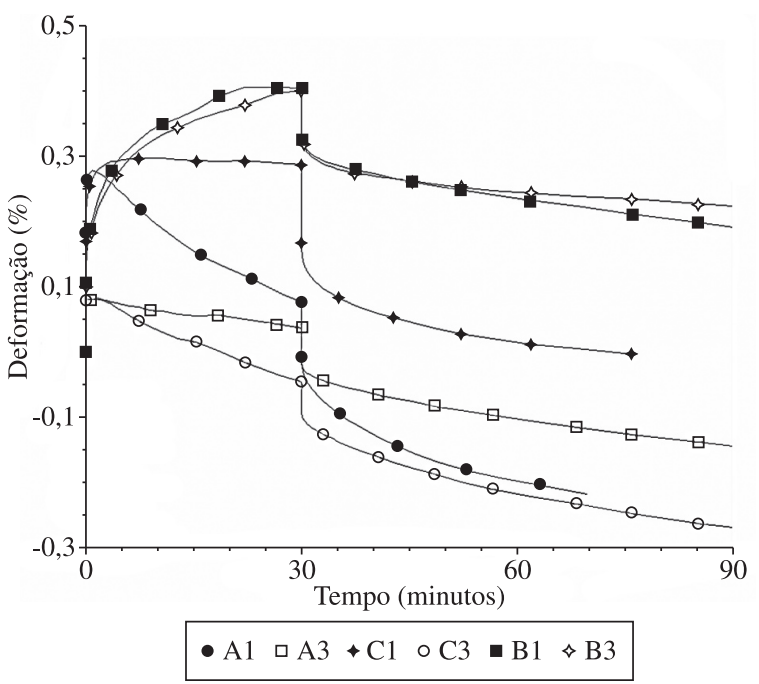

Figura 4. Perfil de deformação de amostras não vulcanizadas e vulcanizadas com diferentes modos de adição do sistema de vulcanização.

\section{Propriedades físico-químicas e mecânicas}

A Tabela 2 apresenta valores de densidade, resíduo de extração seletiva e resistência a tração das amostras compatibilizadas e vulcanizadas. Apenas a amostra B1 (NBR-oxazolina) apresenta uma diferença significativa de densidade. Este fato pode ser consequência de um processo mais eficiente de compatibilização e/ou reticulação, dado confirmado pelo resíduo de extração em tolueno.

A amostra compatibilizada com grupos carboxila (B3) não obteve a mesma eficiência no processo de reticulação, ocorrendo uma perda de massa considerável com a extração em tolueno, seletiva para a fase NBR não vulcanizada. O sistema de compatibilização pode estar influenciando diretamente no processo de reticulação. Ocorrências como esta são encontradas na literatura ${ }^{[24,25]}$. O teor de resíduo de extração da amostra C3 apoia essa hipótese, uma vez que a adição prévia do sistema de reticulação aumentou a eficiência do processo de vulcanização.

Nos resultados de resistência a tração, é possível observar que não existem diferenças significativas de tensão na ruptura, mas o alongamento e o módulo das amostras sem adição prévia do sistema de vulcanização ao MBNBR (amostras B) são superiores, apesar da menor eficiência no processo de reticulação.

\section{Microscopia eletrônica}

A Figura 5 apresenta as imagens de microscopia das amostras C1 e B1 extraídas em tolueno, solvente seletivo para NBR não vulcanizada. Observam-se grandes domínios de NBR extraídos durante a fratura da amostra C1, enquanto que a amostra B1 apresenta menos domínios extraídos devido à fratura, e com tamanhos menores e mais bem dispersos desses domínios. O tamanho da partícula elastomérica dispersa na matriz termoplástica explica a resistência a tração mais pobre da amostra com sistema de reticulação previamente adicionado. Os domínios maiores também são responsáveis por diminuir o módulo. A dispersão de domínios menores gera uma rede mais eficiente em retardar e restringir o movimento das cadeias poliméricas da matriz ${ }^{[22,26]}$. Esse comportamento reflete o comportamento observado para a recuperação da deformação durante o ensaio de fluência é refletido nos melhores resultados de resistência à tração e fluência, mostrando que os resultados são concordantes.

Tabela 2. Propriedades físico-quimicas e mecânicas de amostras compatibilizadas.

\begin{tabular}{lccccc}
\hline & $\begin{array}{c}\text { Densidade } \\
\left(\mathbf{g} / \mathbf{c m}^{\mathbf{3}}\right) \pm \mathbf{3 \%}\end{array}$ & $\begin{array}{c}\text { Resíduo de extração } \\
\mathbf{e m} \text { tolueno }(\boldsymbol{\%}) \pm \mathbf{3 \%}\end{array}$ & $\sigma(\mathbf{M P a})$ & \multicolumn{2}{c}{ Resistência a tração } \\
\cline { 3 - 6 } & 1,747 & 99,9 & $13,6 \pm 0,3$ & $\mathbf{E}(\mathbf{M P a})$ \\
\hline B1 & 1,027 & 100 & $13,3 \pm 0,3$ & $117 \pm 12$ & $118,8 \pm 20$ \\
C1 & 1,019 & 89,2 & $12,2 \pm 0,8$ & $163 \pm 10$ & $306,3 \pm 53$ \\
B3 & 1,017 & 94,3 & $13,2 \pm 0,9$ & $143 \pm 12$ & $136,4 \pm 50$ \\
C3 & & & & &
\end{tabular}




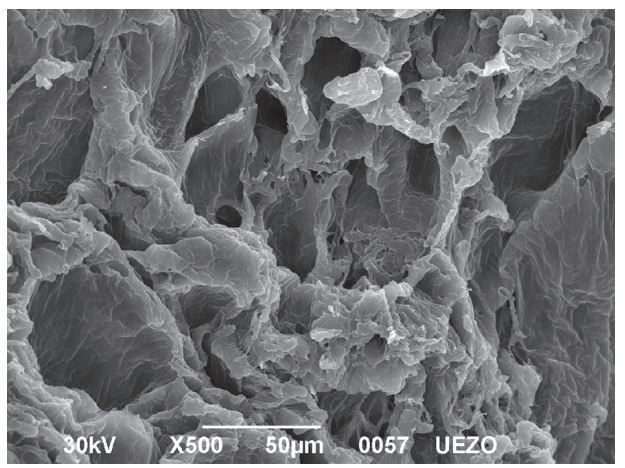

(a)

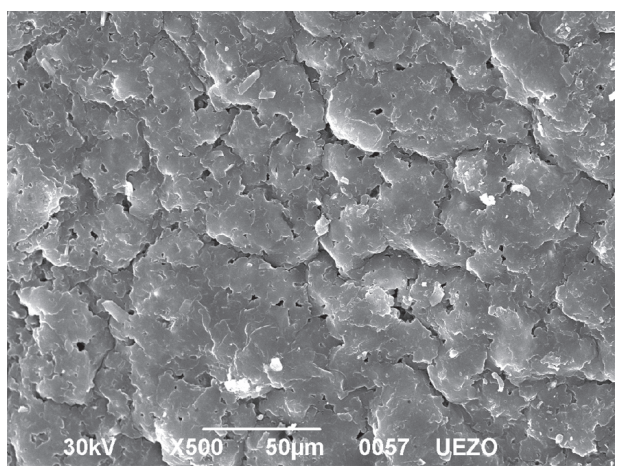

(b)

Figura 5. Imagens de MEV das amostras a) C1; e b) B1.

\section{Conclusões}

O trabalho realizado demonstrou que o teste de fluência pode ser um bom parâmetro de comparação para avaliação da influência de aditivos de processamento e da eficiência de sistemas de compatibilização reativa nas propriedades do material final, tendo boa correspondência com outros ensaios, como resistência a tração, densidade, teor de gel e microscopia eletrônica de varredura. Através da resistência à fluência e recuperação de deformação, foi possível avaliar o efeito dos aditivos no desempenho do material, assim como a escolha de um sistema de compatibilização de acordo com a necessidade de um material com fase elastomérica reticulada ou não. $\mathrm{O}$ EVAMA melhorou a estabilidade dimensional dos corpos de prova de filme. O uso de NBR-carboxilada é a melhor escolha para compatibilização de misturas não vulcanizadas, e a modificação com grupos 2-oxazolina é preferida quando o objetivo é a obtenção de um TPV. A adição do sistema de vulcanização durante o processamento mostrou não reticular completamente a fase NBR, porém apresenta os melhores resultados gerais. Um indicativo do desempenho do material em uma aplicação final de solicitação semelhante pode ser obtido de forma relativamente rápida, prática e com gasto mínimo de material.

\section{Agradecimentos}

Os autores agradecem à CAPES, CNPq e FAPERJ pelo financiamento, à Petroflex, Radici e Crompton pelo material fornecido, e ao Centro Universitário Estadual da Zona Oeste (UEZO) pelas imagens de microscopia eletrônica.

\section{Referências Bibliográficas}

1. Mehrabzadeh, M. \& Buford, R. P. - J. Appl. Polym. Sci., 64, p.1605 (1996).

2. Mehrabzadeh, M. \& Delfan, N. - J. Appl. Polym. Sci., 77, p.2057 (2000).

3. Gessler, A. M.; Haslett Jr., W. H. - U.S. Patent 3037954 (1962).

4. Kumar, R.; George, K. E. \& Thomas, S. - J. Appl. Polym. Sci., 61, p.2383 (1996).

5. Rodgers, B. - "Rubber Compounding: Chemistry and Application". Marcel Dekker Inc., New York (2004).

6. Rocha, T. C. J.; Soares, B. G. \& Coutinho, F. M. B. - Pol.: Ciênc.Tecnol., 17, p.299 (2007).

7. Meyer, A. L.; Souza, G. P.; Oliveira, S. M.; Tomczak, F.; Wasilkoski, C. \& Pinto, C. E. S. - Pol.: Ciênc.Tecnol., 16, p.230 (2006).

8. Coran, A. Y. \& Patel, R. - Rub. Chem. Tech., 53, p.781 (1980).

9. Chowhury, R.; Banerji, M. S. \& Shivakumar, K. - J. Appl. Polym. Sci., 100, p.1008 (2006).

10. Gomes, A. C. O.; Soares, B. G.; Oliveira, M. G.; Oliveira, M. F. L. \& Paranhos, C. M. - e-Polymers. (2009). In press.

11. Bicerano, J. - "Creep, Stress Relaxation, Fatigue and Durability" - in: Prediction of Polymer Properties, cap. 11, J. Bicerano (ed.), Marcel Dekker, Inc., New York (2002).

12. Kolarik, J. \& Pefgoretti, A. - Polym. Test. 27, p.596 (2008).

13. Menard, K. P. - "Dynamic Mechanical Analysis: A practical introduction" - CRC press, New York (1999).

14. Osamnaiye, G. J. \& Adewale, K. P. - Polym. Test., 20, p.363 (2001).

15. Yang, J.; Zhang, Z.; Schlarb, A. K. \& Friedrich, K. - Polymer, 47, p.2791 (2006).

16. El Fray, M. \& Altstadt, V. - Polymer, 44, p.4643 (2003).

17. Almeida, M. S. M.; Soares, B. G. \& Guimarães, P. I. C. - Pol.: Ciênc.Tecnol., 13, p.119 (2003).

18. Rocha, L. A. C.; Schuster, R. H.; Jacob, M. M. \& Samios, D. - Pol.: Ciênc. Tecnol. 14, p.318 (2004).

20. Roguet, E.; Castagnet, S. \& Grandidier, J. C. - Mech. Mat., 39, p.380 (2007).

21. Araújo, E. M.; Hage Jr., E. \& Carvalho, A. J. F. - J. Mat. Sci., 38, p.3515 (2003).

22. Li, T. \& Yan, N. - Composites: Part A., 38, p.01 (2007).

23. Yan, L. T.; Qian, Z. Y.; Guo, B. H.; Xu, J. \& Xie, X. M. - Polymer, 46, p.11918 (2005).

24. Rangaraj, S. V. \& Smith, L. V. - Mech. Time-Dep. Mat., 3, p.125 (1999).

25. Oliveira, M. G. \& Soares, B. G. - Pol.: Ciênc.Tecnol., 12, p.301 (2002).

26. Passador, F. R.; Pessan, L. A. \& Rodolfo Jr., A. - Pol.: Ciênc. Tecnol., 16, p.174 (2006).

Enviado: 20/02/09

Reenviado: 20/04/09

Aceito: $24 / 04 / 09$ 